\title{
Análisis del uso de las herramientas de gestión ambiental en las empresas comerciales del cantón Morona
}

\section{Analysis of the use of environmental management tools in the cantón morona commercial companies}

\author{
López Jara, Ana Alexandra ${ }^{1}$, Calle Samaniego, Dayana Brigiht ${ }^{1}$ y Molina Benalcázar, Aly Midory ${ }^{1}$ \\ ${ }^{1}$ Universidad Católica de Cuenca, Sede Macas, Ecuador \\ *alopezj@ucacue.edu.ec
}

\begin{abstract}
Resumen
El fenómeno de la globalización agregado a los problemas ambientales causados en el entorno por el ser humano y el desarrollo inconsciente de las actividades económicas de las empresas del sector comercial se han convertido en una severa problemática a nivel mundial, lo que ha conducido a las empresas a la adopción voluntaria de herramientas de gestión ambiental con la finalidad de elaborar y ejecutar sus actividades con alta calidad, satisfaciendo las necesidades de los clientes como de las partes relacionadas en materia de medio ambiente. Las herramientas de gestión ambiental constituyen recursos que utiliza la sociedad para llevar a cabo medidas destinadas a solucionar los problemas ambientales. La investigación consistió en realizar un análisis sobre el grado de utilización de herramientas de gestión ambiental en las empresas comerciales del cantón Morona, aplicando como instrumentos de evaluación encuestas para medir el grado de conocimiento, utilización e influencia de las herramientas de gestión ambiental en las actividades económicas de los negocios, con el fin de concientizar su uso para atribuir al desarrollo sostenible. Los resultados demuestran que la mayor parte de las empresas comerciales en el Cantón Morona carecen de conocimientos sobre las herramientas de gestión ambiental, por lo cual no hacen uso de estos instrumentos, sin embargo, parte de las empresas que conocen sobre este tema, han implementado acciones de manera voluntaria para cuidar el medio ambiente como es el reciclaje y recolección de residuos, el cual ha influido significativamente en sus actividades económicas.
\end{abstract}

Palabras clave: Medio Ambiente, Desarrollo Sostenible, Herramientas de gestión ambiental..

\begin{abstract}
The phenomenon of globalization added to the environmental problems caused in the environment by the human being and the unconscious development of the economic activities of the companies of the commercial sector have become a severe problem worldwide, which have led companies To the voluntary adoption of environmental management tools with the purpose of elaborating and executing their activities with high quality, satisfying the needs of customers and related environmental parties. Environmental management tools are resources that society uses to carry out measures aimed at solving environmental problems. The research consisted of an analysis of the degree of use of environmental management tools in the commercial enterprises of the canton of Morona, applying as an evaluation tool surveys to measure the degree of knowledge, use and influence of the environmental management tools in the activities Economic aspects of business, in order to raise awareness of its use to be attributed to sustainable development. The results show that most of the commercial companies in the Canton of Morona lack knowledge about the environmental management tools, so they do not make use of these instruments. However, some companies that know about this subject have implemented actions of Voluntary way to take care of the environment such as recycling and waste collection, which has significantly influenced their economic activities.
\end{abstract}

Key words: Environment, Sustainable Development, Environmental management tools..

\section{Introducción}

Los impactos ambientales provocados en mayor parte por la sobreexplotación de los recursos naturales y el inapropiado uso del ambiente por parte del ser humano y las industrias, han conllevado a las empresas a reflexionar e implementar herramientas de gestión ambiental para reducir en gran medida la contaminación.
Estas herramientas han contribuido considerablemente a que las empresas aumenten su competitividad empresarial de manera sostenible, aumentando los niveles de calidad de vida, en el aspecto ambiental, económico y social.

El Ecuador contribuyendo con el medio ambiente ha incorporado principios medioambientales en su Constitución política de la República del Ecuador, como también ha creado normativas e instituciones centradas en la gestión 
medioambiental como es la Ley de prevención y control de la contaminación ambiental y Ley de Gestión Ambiental y el ente regulador como el Ministerio del Ambiente.

El presente trabajo investigativo está enfocado en medir el grado de conocimiento, utilización e influencia de las herramientas de gestión ambiental en las actividades económicas de las empresas comerciales del cantón Morona, con el fin de concientizar su uso para atribuir al desarrollo sostenible.

\section{Marco téorico}

\subsection{Evolución del impacto ambiental}

El impacto del ser humano en el ambiente surge desde epocas muy antiguas, aproximadamente por el siglo XI se manifestaba un efecto en aguas contaminadas provocando enfermedades como cólera, disentería entre otras. Sin embargo, con la llegada de la Evolucion Industrial agrabaron mas los problemas ambientales, principalmente en el aire y agua en localidades que en aquella época no contaban con las capacidades suficientes para corregirlos.

Asi mismo, (Cordero, 2004) afirma que el problema medioambiental surge desde un tiempo muy ambiguo, aproximadamente por el año 1962, en donde se publicó el libro del autor Rachel Carson de Silent Spring. En el cual el autor hace enfasis al cambio del uso de pesticidas que en aquella epoca fueron manejados para la agricultura, puesto que los mismos provocaban grandes daños para la salud del ser humano y de los animales.

Años despues, aproximadamente en 1968, UNESCO presenta un foro de diálogo e intercambio de conocimientos para la conceptualización de desarrollo sostenible, el cual se refiere a un desarrollo que permite satisfacer la necesidad acutal sin comprometer o perjudicar la posibilidad de la futura generación.

Así tambien, en 1969 Estados Unidos aprueba un acta de política Nacional Ambiental, con lo que más tarde se crea la primera agencia nacional para la protección del medio ambiente, misma que pertenece al gobierno federal de los Estados Unidos, cuyo objetivo es la protección de la salud del ser humano y protección del ambiente en aspectos como el suelo, agua y aire.

De igual forma en 1970 se desarrolla el primer "Día de la tierra" el cual fue promovido por el senador Gaylord Nelson, donde se deduce que asistieron millones de personas con el fin de concientizar sobre problemas, producto de la gran contaminación en el medio ambiente y la conservación del mismo. A finales de ese año se presentan catástrofes ambientales como son el derrame petróleo de Amoco en Cádiz y la fuga en el reactor nuclear en la isla Three Mile.

Después de haber transcurrido dos años del anterior acontecimiento en 1972 se desarrolla la conferencia de las Naciones Unidas en Estocolmo "Human Enviroment" (Ambiente Humano), la cual conlleva al desarrollo de agencias de protección al ambiente, así como un programa ambiental de las Naciones Unidas (UNEP)
"Posteriormente en 1982 las Naciones Unidas adoptaron la convención de la ley del mar, en donde se establece las reglas referentes a las normas ambientales, así como aspectos relacionados con la contaminación al ambiente marino" (Cordero, 2004).

Esta ley fue aprobada despues de nueve y largos años de trabajo,considerandose como uno de tratados a nivel internacional mas importante en historia. Por tanto esta norma ha permitido la protección del ecosistema del ambiente marino.

Por otro lado, (Cordero, 2004) menciona, que 1992 se desarrolla la conferencia de las Naciones Unidas en el Ambiente y Desarrollo en Rio de Janeiro, de la cual tiene como resultado la publicación de la Agenda 21, misma que fue firmada y aprobada por 173 gobiernos. Establece principios de desarrollo sostenible que son aplicables internacionalmente cuyo propósito es mejorar la calidad de vida y el medio ambiente de todos los habitantes.

Finalmente en el año 2002 se llevó a cabo la cumbre mundial en desarrollo sostenible, cuyo objetivo fue el de hacer una declaración pública en pro del desarrollo sostenible, trabajar en planes de acción en áreas de agricultura, biodiversidad, energía, agua, saneamiento ambiental y promover alianzas entre los países, organismos no gubernamentales e internacionales.

De lo presentado anteriormente, se analiza que el daño al medio ambiente es una de las grandes problemáticas potenciales que han existido desde épocas muy antiguas y ha generado grandes afectaciones hasta la actualidad. Por tanto, en busca de dar solución a este acontecimiento, existen países que han implementado dentro de su legislación ambiental normas y leyes que permitan reducir y controlar el impacto ambiental, sensibilizando, concientizando y promoviendo un desarrollo sostenible.

\subsection{La gestion Ambiental en el Ecuador}

Es así que el Ecuador al igual que otros paises en vias de desarrollo, no se ecuentra exento de los efectos provocados por el ser humano en el medio ambiente.

Por ello, para controlar las secuelas del daño ambiental, el Ecuador ha incorporado principios medioambientales en su Constitución política República del Ecuador ejecutado por la Asamblea Nacional Constituyente del año 2008. Por otro lado, en su legislación ambiental existen leyes y normativas medioambientales, que regulan la seguridad y conservación del medio ambiente, entre las que se puede mencionar, la Ley de Preservación y Control de la Contaminación Ambiental, la Ley de Gestión Ambiental y el ente regulador que se denomina al Ministerio del Ambiente. Las cuales se describen a continuación.

De lo mencionado anteriormente, la Ley de prevención y Control ambiental, fue la primera ley instaurada en materia ambiental en el año 1976, mediante Decreto Ejecutivo No. 374 el 31 de mayo. La cual dicta medidas encaminadas a la prevención y control de la contaminación del suelo, agua y aire. 
Asi mismo, el 4 de octubre de 1996 mediante Decreto Ejecutivo No. 195 publicado en el Suplemento- Registro Oficial No. 40, se estableció el Ministerio del Ambiente, como el máximo ente regulador de la Gestión Ambiental en el Ecuador; encargado de garantizar un ambiente sano y ecológicamente equilibrado, promoviendo el desarrollo sustentable, conservando su biodiversidad, mejorando su calidad ambiental y reconociendo al agua, aire y suelo como recursos naturales estratégicos (Tecnologias limpias, 2007)

De igual manera, el 30 de julio de 1999 mediante Decreto Ejecutivo No. 1761, publicado en el SuplementoRegistro Oficial No. 245, se promulgó la Ley de Gestión Ambiental, misma que establece los principios y directrices de política ambiental; las obligaciones, responsabilidades, niveles de participación de los sectores público y privado en la gestión ambiental y señala los límites permisibles, controles y sanciones en esta materia. Es así que esta ley regula todas las actividades relacionadas al medio ambiente del estado ecuatoriano (Ley de gestión ambiental, 2012)

Finalmente, se establece la Constitución Política del Ecuador ejecutada por la Asamblea Nacional Constituyente en el año 2008, que en su contenido se exponen artículos enfocados a la protección, control y cuidado del medio ambiente, de los cuales se hace mención al siguiente artículo; (Marco Normativo Ambiental, s.f.)

Según el artículo 14 de la Constitución del Ecuador, del capítulo segundo- sobre Derechos del buen vivir Sección segunda-Ambiente sano, explica que se reconoce el derecho de la población a vivir en un ambiente sano y ecológicamente equilibrado, que garantice la sostenibilidad y el buen vivir, sumak kawsay

Así también se declara de interés público la preservación del ambiente, la conservación de los ecosistemas, la biodiversidad y la integridad del patrimonio genético del país, la prevención del daño ambiental y la recuperación de los espacios naturales degradados (Marco Normativo Ambiental, s.f.)

En virtud de lo expuesto, las normativas y leyes que se encuentran establecidas en la legislación ambiental del estado ecuatoriano, han dictado medidas que permiten controlar y prevenir la contaminación del suelo, aire y agua, así como también la sobre explotación de recursos naturales y conservación del medio ambiente y su ecosistema, dando así, derecho a una mejor calidad de vida al ciudadano. Sin embargo para garantizar el cumplimiento de las mismas y un desarrollo sustentable en el medio ambiente, deben ser ejecutadas por el Estado y la sociedad en conjunto.

\subsection{Herramientas de Gestión ambiental}

A más de las normativas regulatorias sobre el medio ambiente, dentro de la Gestión Ambiental, se abarcan herramientas de gestión ambiental que pretenden hacer énfasis en el mejoramiento del desempeño ambiental de las empresas, promoviendo así el desarrollo sustentable. Entre las cuales se pueden mencionar las siguientes:
- Sistema de Gestión Ambiental.

- Certificaciones Ambientales.

- Etiquetado Ecológico.

- Auditorías Ambientales.

- Análisis del Ciclo de Vida.

En relación a lo expuesto, un sistema de gestión ambiental es aquella parte del sistema general de gestión que comprende la estructura organizativa, las responsabilidades, las prácticas, los procedimientos, los procesos y los recursos para determinar y llevar a cabo la política ambiental dentro de una empresa(Massolo, 2015)

Es así que un sistema de gestion ambiental actua como una herramienta que puede ser implementada dentro de una organización de manera voluntaria sea publica o privada, cuya finalidad es mejorar el comportamiento ambiental de la organización, cumpliendo con su politica, objetivos y metas ambientales.

Así mismo, (Montiel, 2015) menciona que un buen sistema de gestion ambiental deberá contener:

- Definida una política ambiental.

- La planificacion y establecimientos de objetivos de mejora.

- Acciones preventivas y correctivas.

- Revision del sistema de gestión de calidad

- Validación o certificación del SGA por una entidad acreditada

- Desarrollo de tecnologias limpias.

Por otro lado, las certificaciones ambientales constituyen una herramienta enlazada al Sistema de Gestión Ambiental implantado por la empresa, garantizando que el mismo sea eficaz y de calidad (Ortiz, 2009)

Estas certificaciones pueden ser emitidas por entidades certificadoras internacionales como la Organización Internacional para Normalización ISO

De las cuales se puede mencionar la certificación de la norma ISO 14001, que se encuentra vinculada específicamente con el sistema de gestión de calidad. La cual es aplicable en todos los sectores de la industria u organizaciones del sector público o privado, independientemente de su tamaño o ubicación geográfica.

Para la certificación de la norma ISO 14001, la empresa debe cumplir con los principales requisitos de esta norma, tales como crear un plan de manejo ambiental, en el cual se describan políticas, objetivos y metas ambientales, establecer procedimientos que permitan cumplir esas metas. Así como también responsabilidades que estén definidas, actividades de capacitación al personal y un sistema que permita controlar cambios y avances realizados.

De igual manera, el reglamento Comunitario de Ecogestión y Ecoauditoría por sus siglas (EMAS), es una normativa voluntaria de la Unión Europea que reconoce a aquellas organizaciones que han implementado un sistema de gestión ambiental (SGA) y han adquirido un compromiso de mejora continua, del cual la organización da cuenta periódicamente de su funcionamiento mediante una declaración ambiental verificada por auditorías independientes. 
Cabe recalcar que la Norma ISO 14001 sobre sistemas de gestion ambiental, es una norma que puede ser aplicada internacionalmente, la cual certifica a una organización independientemente sea esta publica o privada, el cumplimiento de los requisitos principales de dicha norma, como el establecimiento de politicas, objetivos y metas medioambientales, que permiten lograr a las empresas un equilibrio ambiental.

Asi mismo la cerficación de esta norma puebe brindar ventajas a para las empresas tales como:

- El mejoramiento de la imagen y reputacion de la misma

- La apertura, expansión y oportunidad de crecimiento en mercados internacionales.

- Acoger mayor clientes que se sensibilicen ante la proteccion del medio ambiente

- Aseguramiento del cumplimiento de requisitos legales relacionados con temas ambientales.

Mientras que el reglamento Comunitario de Ecogestión y Ecoauditoría (EMAS) es aplicable solo en la Union Europea, por tanto reconoce aquellas empresas u organizaciones que de carácter voluntario han implementado un sistema de gestion ambiental en base al cumplimiento de los requisitos que dicta este reglamento.

Por otro lado, se describe el etiquetado ecológico, el cual es otra de las herramientas de gestión ambiental, adoptado por las empresas de fabricación, como un distintivo a través del cual se identifica que el producto ha sido elaborado con el menor impacto posible en el medio ambiente(Massolo, 2015)

Esta herramienta representa un instrumento de gran utilidad para la empresa, para promover el diseño, producción y comercialización de productos de menor impacto ambiental y con los niveles necesarios de calidad y seguridad. Mientras que para los consumidores brinda información que les permita escoger y usar productos menos perjudiciales para el ambiente.(Massolo, 2015)

De igual manera la auditoría ambiental conforma otro de los instrumentos de gestión ambiental, que representa una de las herramientas más utilizadas dentro de una empresa, ya que permite realizar una evaluación periódica, sistemática y documentada de la eficiencia y eficacia de la organización, así como también del sistema de gestión ambiental y del cumplimiento de las normativas ambientales.

Así mismo, el análisis del ciclo de vida, es una herramienta que se maneja en las empresas con el fin de determinar el impacto ambiental que produce un producto desde el inicio hasta el final de todas las fases de su vida útil, con el fin de mejorarlo para reducir su impacto. Este análisis trata de identificar y caracterizar los diferentes impactos ambientales que se generan en un producto no solo en la fabricación sino también en otras fases como desde la adquisición de la materia prima para elaboración del mismo. En si el análisis del ciclo de vida de un producto, plantea manejar los residuos en forma sustentable, buscando una mejora continua en sus procesos.
Argumentada la investigación y en concordancia a lo tratado anteriormente, se deriva el objetivo general de este trabajo, que consiste en evaluar el análisis y uso de las herramientas de gestión ambiental en las empresas comerciales del cantón Morona.

\section{Metodología}

Esta investigación es de carácter descriptivacuantitativa, debido a que la misma permitirá recopilar información para conocer el grado de conocimiento, utilización e influencia de las herramientas de gestión ambiental en cada una de las empresas comerciales del cantón Morona.

Para la recolección de la información se aplicaron encuestas dirigidas a los propietarios de las empresas comerciales del Cantón Morona, tomando como muestra 264 empresas comerciales, de un universo de 12600.

\section{Resultados}

Los resultados de la aplicación de encuestas, dirigidas a los propietarios de las 263 empresas comerciales en el Cantón Morona, se presentan a continuación evaluados por componentes:

\subsection{COMPONENTE: DATOS GENERALES}

\subsection{1 ¿Tipo de actividad económica a la que se dedica la empresa \\ $?$}

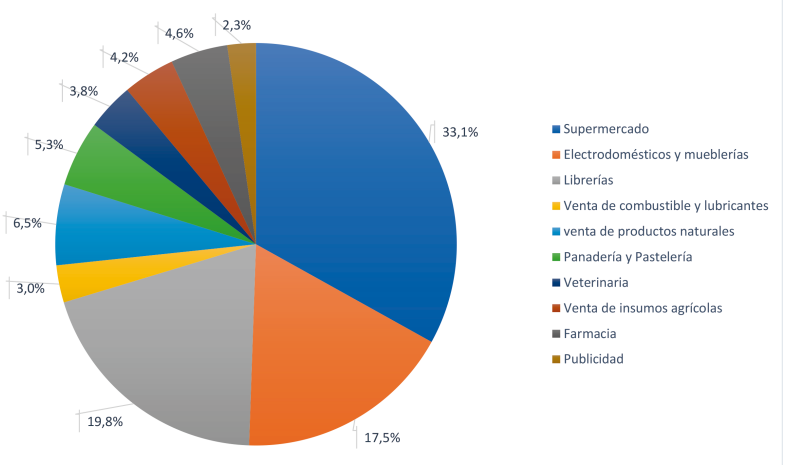

Figura 1. Tipo de actividad economica

Fuente: Encuestas aplicadas en las empresas comerciales del cantón Morona.

Los resultados obtenidos según el componente "DATOS GENERALES", de la encuesta aplicada a las 263 empresas comerciales del cantón Morona, deduce que entre las diferentes actividades económicas a las que se dedican las empresas comerciales están las siguientes: publicidad, venta de lubricantes y combustibles, veterinaria, venta de insumos agrícolas, farmacia, panadería, venta de productos naturales, electrodomésticos y mueblería, librerías y supermercados. Del cual la actividad comercial en supermercados sobresale con un valor equivalente al $33.1 \%$, mientras 
que la menor actividad comercial desarrollada en el cantón se muestra en la publicidad, reflejando así el $2.3 \%$. Por otro lado el $64.6 \%$ restante corresponde a las actividades comerciales anteriormente citadas.

\subsection{COMPONENTE: RESPONSABILIDAD AMBIEN- $T A L$}

4.2.1 Desarrolla acciones para el cuidado del medio ambiente?

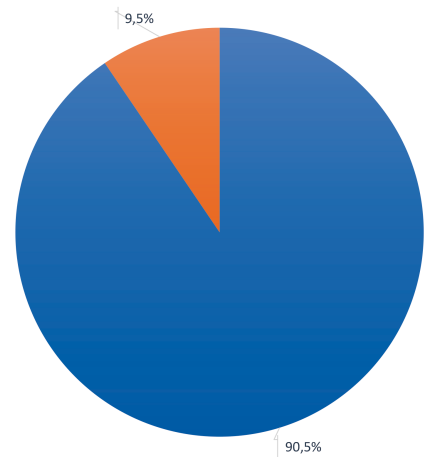

asi
ano

Figura 2. Implementacion de acciones para el cuidado medioambiental

Fuente: Encuestas aplicadas en las empresas comerciales del cantón Morona.

De los resultados obtenidos en el componente "RESPONSABILIDAD AMBIENTAL", el 9.5\% desarrolla acciones en beneficio al medio ambiente, implementando herramientas y políticas que ayudan a contrarrestar estos efectos, mientras que el $90.5 \%$ de las empresas comerciales en el Cantón Morona, no han desarrollo medidas para combatir el daño e impacto ambiental, esto debido a ciertos factores como son: la ausencia de conocimientos sobre aspectos medioambientales, y la falta de concientización en el cuidado, protección y conservación del ambiente y ecosistema en el sector comercial.

\subsection{COMPONENTE: CONOCIMIENTO Y UTILIZA- CION DE LAS HERRAMIENTAS DE GESTION AM- BIENTAL}

\subsection{1 ¿Conoce Ud. Sobre las herramientas de Gestión ambiental?}

Del componente conocimiento y utilización de las herramientas de gestión ambiental se obtuvieron los siguientes resultados (Figura 3): El 74,9\% de las empresas comerciales en el cantón Morona, carecen de conocimientos sobre herramientas de gestión ambiental; esto se debe a la falta de difusión y comunicación por parte de las autoridades responsables en materia medioambiental, o la vez por el desinterés de los propietarios de las empresas. Así mismo el $25,1 \%$ de las entidades demuestra tener conocimiento respecto al tema, es por ello que han implementado acciones que permitan contrarrestar el impacto ambiental.

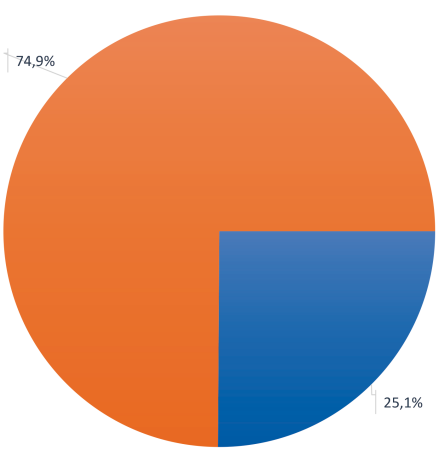

Figura 3. Nivel de conocimiento de las herramientas de gestión ambiental

Fuente: Encuestas aplicadas en las empresas comerciales del cantón Morona.

\subsubsection{En caso de ser afirmativo, ¿Las ha implementado?}

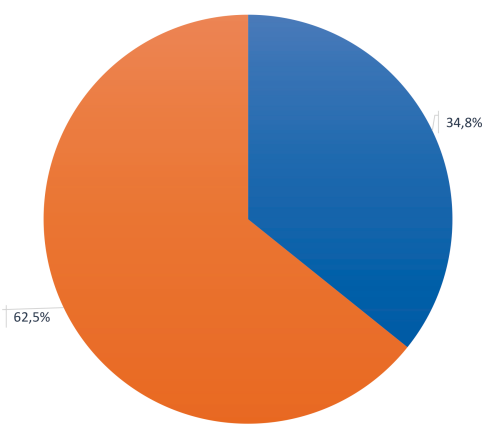

Figura 4. Implementacion de las herramientas de gestión ambiental

Fuente: Encuestas aplicadas en las empresas comerciales del cantón Morona.

Al analizar el siguiente grafico correspondiente al mismo componente, se deduce que del $25,1 \%$ de empresas del cantón Morona que poseen conocimiento en el tema de herramientas de gestión ambiental, el 34,8\% ha implementado dichos instrumentos de manera voluntaria para contribuir al cuidado del medio ambiente y el 65,2\% restante no las han adaptado. Este resultado es producto de la falta de concientización respecto a temas ambientales.

\subsubsection{Cuál de las siguientes herramientas de gestión am- biental utiliza?}

De las 23 empresas, que representan el 34,8\% de las cuales han decidido por implementar las herramientas de gestión ambiental, se concluye que el principal instrumento, en mayor parte utilizado por las empresas del cantón Morona para contribuir al cuidado del medio ambiente es el reciclaje de residuos, que hace referencia a la opción “OTROS” del gráfico analizado(gráfico 5). Además se presentan el $17,4 \%$ en certificaciones, el $13 \%$ Auditorías 
ambientales, $13 \%$ análisis del ciclo de vida, 4,3\% etiquetado ecológico y el 4,3\% en sistemas de gestión ambiental, mismas que son aplicadas de manera voluntaria, garantizando una mejora continua en el procesos de la actividad comercial de las empresas con el fin de contrarrestar el impacto sobre el medio ambiente.

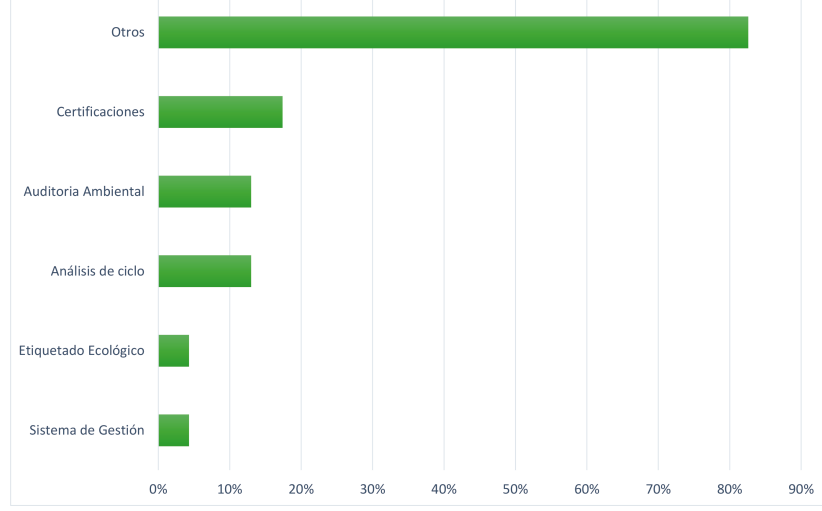

Figura 5. Tipo de herramientas de gestión ambiental utilizada

Fuente: Encuestas aplicadas en las empresas comerciales del cantón Morona.

\subsection{4 ¿Cuál ha sido los beneficios que ha obtenido me- diante la aplicación de las distintas herramientas de Gestión ambiental}

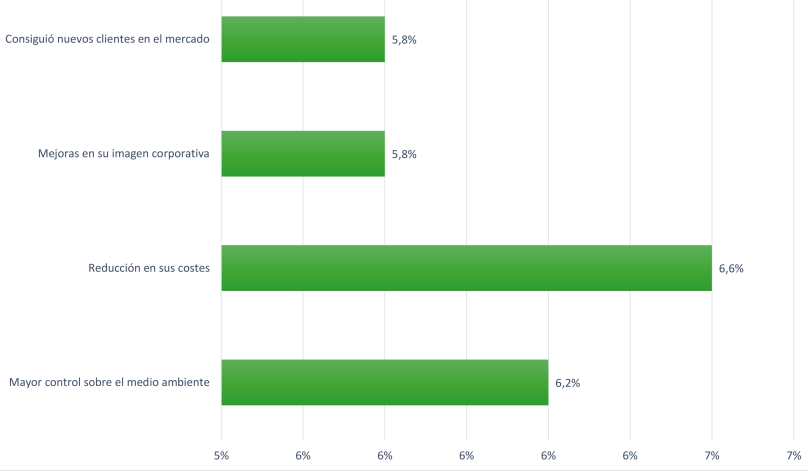

Figura 6. Beneficios de la implementación de las herramientas de gestión ambiental

Fuente: Encuestas aplicadas en las empresas comerciales del cantón Morona.

La implementación de las herramientas de Gestión Ambiental en las empresas comerciales del cantón Morona, han conllevado los siguientes beneficios (gráfica 6): en un $6,2 \%$ las empresas adquirieron un mayor control sobre el medio ambiente, el 6,6\% obtuvo reducción en sus costes, mientras que el 11,6\% obtuvo mejoras en su imagen corporativa, y consiguió nuevos clientes en el mercado.

\subsection{COMPONENTE: PRÁCTICAS DE CUIDADO AM- BIENTAL}

\subsection{1 ¿Posee la empresa una política medioambiental?}

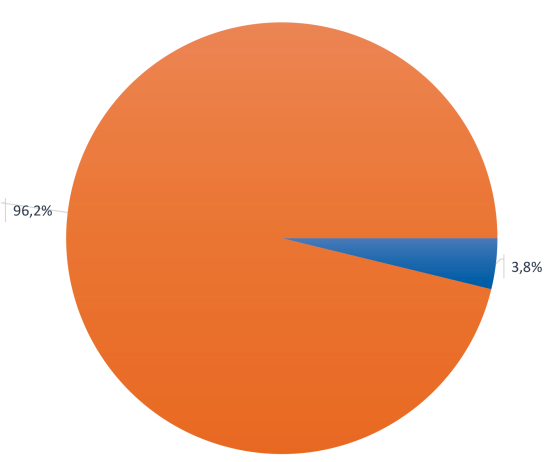

Figura 7. Implementacion de una política ambiental

Fuente: Encuestas aplicadas en las empresas comerciales del cantón Morona.

De la interpretación gráfica 7 , se demuestra el 3,8\% de empresas comerciales del cantón Morona posee una política medioambiental, mientras que el 96,2\% carecen de políticas medioambientales, ya sea por falta de conocimiento sobre las distintas herramientas de gestión ambiental y a su vez por la ausencia de implementación de acciones que permitan contrarrestar la contaminación al medio ambiente.

\subsection{2 ¿Participa en alianzas con otras organizaciones desarrollando acciones en favor del cuidado del medio ambiente?}

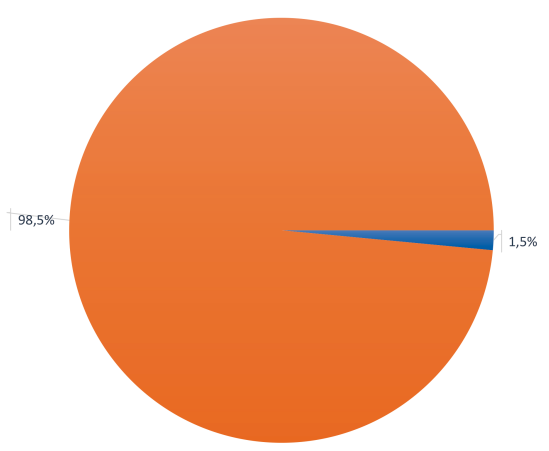

- $\mathrm{Si}$
No

Figura 8. Alianzas emprendidas para el cuidado del medio ambiente

Fuente: Encuestas aplicadas en las empresas comerciales del cantón Morona.

Al examinar los resultados de la siguiente representación gráfica (gráfica 8) se determina que el $1.5 \%$ de empresas comerciales en el cantón Morona, que han desarrollado acciones en beneficio a la protección y conservación 
medio ambiental, se han aliado a distintas instituciones públicas como son al Ministerio de Agricultura, Ganadería, Acuacultura y Pesca (MAGAP) y al Gobierno Autónomo Descentralizado del cantón Morona, mediante convenios y desarrollo de ferias de concientización en temas medioambientales para la ciudadanía. Mientras que el $98.5 \%$ restante no han implementado acciones para el cuidado del medio ambiente.

\section{Conclusiones}

Con el desarrollo del presente trabajo de investigación, se ha logrado conocer cuál es el nivel de conocimiento, utilización e influencia de las herramientas de gestión ambiental en cada una de las empresas comerciales del cantón Morona.

El termino herramientas de gestión ambiental se refiere a un conjunto de acciones que desarrollan las empresas, con el fin de reducir el impacto ambiental causado en el medio ambiente y garantizar el desarrollo sostenible.

Del análisis de resultados obtenidos en la investigación, se deduce que, en el sector comercial del Cantón Morona, gran parte de las empresas desconocen sobre las herramientas de gestión ambiental, lo cual ha repercutido en su no utilización. Esto se debe entre otros motivos a la falta de difusión de temas de protección ambiental, por parte de la entidad encargada en materia de medio ambiente, además por la carencia de concientización de los representantes de las empresas comerciales que no respetan al entorno en que se habita, por último, por el desinterés de abrirse a un cambio cultural y de tomar medidas preventivas que contribuyan a la mejora del medio ambiente y de la supervivencia del ser humano.

Así mismo existen ciertas empresas que en un menor porcentaje poseen conocimiento sobre las herramientas de gestión ambiental y por ende han implementado acciones sobre el tema, de las cuales se puede destacar una de las empresas dedicada a actividades agropecuarias, que ha aplicado las herramientas de gestión ambiental citadas en la presente investigación, siendo así reconocida y certificada por organismos autorizados como la Organización Internacional para Normalización ISO- norma ISO 14001, enfocada a un sistema de gestión ambiental. Por otra parte tambien se ha promovido el reciclaje y clasificación de residuos por parte de la minoría de las empresas que poseen conocimientos ambientales, siendo estas conscientes de los efectos secundarios que conlleva la contaminación en nuestro medio.

La implementación y utilización del reciclaje de residuos como herramienta de gestión ambiental en las empresas comerciales del Cantón Morona, ha influido positivamente, proporcionando serie de ventajas como un mayor control sobre la situación ambiental, la reducción de costes, la mejora de la imagen corporativa y la atracción de nuevos clientes al mercado.

Finalmente se puede concluir que en las empresas comerciales del Cantón Morona, existe poca concientización hacia el cuidado del medio ambiente, debido a que en las mismas solo se ha empeñado en obtener lucro, dejando desapercibido cualquier afectación que pueda provocar el desarrollo de su actividad comercial en nuestro entorno. Por tal motivo es necesario que las empresas reflexionen y desempeñen acciones de manera voluntaria para la protección de nuestro ecosistema, siendo este el mejor legado que puedan recibir las futuras generaciones.

\section{Referencias Bibliográficas}

Cordero, M. B. (2004). Gestion Ambiental Camino al desarrollo sostenible. Euned.

Ley de gestión ambiental. (2012). Descargado de http://www.ambiente.gob.ec/ wp-content/uploads/downloads/2012/ $09 /$ LEY-DE-GESTION-AMBIENTAL.pdf

Marco Normativo Ambiental. (s.f.). Descargado de https://sites.google.com/site/ marconormativoambiental / ecuador

Massolo, L. (2015). Introduccion a las herramientas de gestion ambiental. Edulp.

Montiel, M. (2015). Propuesta de un Sistema de Gestion Ambiental basado en la Norma ISO 14001 Para Industrial Pesquera Santa Priscila S.A.

Ortiz, L. M. (2009). Normas ISO 14000 como intrumento de gestion ambiental empresarial (Inf. Téc.). Veracruz.

Tecnologias limpias. (2007).

Aceptado: 9 de noviembre de 2017 
